Meta

Journal des traducteurs

Translators' Journal

\title{
Quelques principes de normalisation des noms botaniques français : les arbres d'Amérique du Nord
}

\section{Miroslav M. Grandtner et Marc-Alexandre Beaulieu}

Volume 55, numéro 3, septembre 2010

URI : https://id.erudit.org/iderudit/045077ar

DOI : https://doi.org/10.7202/045077ar

Aller au sommaire du numéro

Éditeur(s)

Les Presses de l'Université de Montréal

ISSN

0026-0452 (imprimé)

1492-1421 (numérique)

Découvrir la revue

Citer cet article

Grandtner, M. M. \& Beaulieu, M.-A. (2010). Quelques principes de normalisation des noms botaniques français : les arbres d'Amérique du Nord. Meta, 55(3), 558-568. https://doi.org/10.7202/045077ar
Résumé de l'article

Le présent article porte sur la normalisation des noms botaniques français désignant les arbres indigènes d'Amérique du Nord. Il propose une nomenclature normalisée française, inspirée des noms scientifiques latins et des noms populaires français. Ses quatre principes de base sont : le caractère binomial du nom, son exclusivité, l'exactitude de sa signification botanique et son universalité. Sont également analysés d'autres principes comme la priorité du nom existant sur le néonyme ainsi que le recours aux grandes catégories conceptuelles, telles que la morphologie, la ressemblance avec d'autres taxons, la toponymie, l'écologie, l'anthroponymie et l'utilisation de la plante. Enfin, un aperçu du travail qui reste à faire pour normaliser la totalité des noms français de la dendroflore mondiale vient conclure l'article. 


\title{
TERMINOLOGIE ET LINGUISTIQUE
}

\section{Quelques principes de normalisation des noms botaniques français: les arbres d'Amérique du Nord}

\author{
MIROSLAV M. GRANDTNER \\ Université Laval, Québec, Canada \\ miroslav.grandtner@sbf.ulaval.ca
}

MARC-ALEXANDRE BEAULIEU

Gouvernement du Canada, Gatineau, Canada

marc-alexandre.beaulieu@tpsgc-pwgsc.gc.ca

\section{RÉSUMÉ}

Le présent article porte sur la normalisation des noms botaniques français désignant les arbres indigènes d'Amérique du Nord. Il propose une nomenclature normalisée française, inspirée des noms scientifiques latins et des noms populaires français. Ses quatre principes de base sont: le caractère binomial du nom, son exclusivité, l'exactitude de sa signification botanique et son universalité. Sont également analysés d'autres principes comme la priorité du nom existant sur le néonyme ainsi que le recours aux grandes catégories conceptuelles, telles que la morphologie, la ressemblance avec d'autres taxons, la toponymie, l'écologie, l'anthroponymie et l'utilisation de la plante. Enfin, un aperçu du travail qui reste à faire pour normaliser la totalité des noms français de la dendroflore mondiale vient conclure l'article.

\begin{abstract}
In this article, the practice and challenges of the standardization of French botanical names are assessed by comparing names from different nomenclatures that refer to the native trees of North America. A French standardized nomenclature is proposed, taking into account both the Latin scientific nomenclature and the French vernacular nomenclature. The following basic principles regarding name formation are first analyzed: the binomial nature of the name, its exclusivity, the correctness of its botanical meaning and its universality. Complementary principles such as the priority of an existing name over a newly formed one, and the application of major conceptual categories, such as morphology, similarity with another species, toponymy, ecology, anthroponymy and the use of the species, are also taken into consideration. Finally, an insight into the work that remains to be done in order to standardize the French species names for the whole tree flora of the world concludes this article.
\end{abstract}

\section{MOTS-CLÉS/KEYWORDS}

normalisation, noms botaniques français, arbres, Amérique du Nord standardization, French botanical names, trees, North America 


\section{Introduction}

Nommer les plantes est une préoccupation très ancienne (Pajaud 1989; Linnaeus 2003), en grande partie liée au fait que le nom attribué à une plante ne peut en soi évoquer qu'une seule des nombreuses caractéristiques de celle-ci (un nom, un concept, une espèce). La terminologie populaire, qui procède souvent par métaphores, est antérieure à la terminologie scientifique, qui repose essentiellement sur la taxonomie (Bizet et Walter 2008). L'aspect utilitaire de la terminologie populaire a souvent conduit à une multiplication débridée des noms, avec pour cas extrême le gommier rouge (Bursera simaruba), qui est désigné par 19 noms français, 32 noms anglais, 99 noms espagnols, 37 noms d'autres langues et 4 noms commerciaux, soit 181 noms populaires différents (Grandtner 2005). À cela s'ajoutent de nombreuses erreurs botaniques: le mot 'cèdre' (Cedrus) peut désigner six genres différents et le sapin traînard n'est pas un sapin (Abies) mais un if, l'if du Canada (Taxus canadensis). D’où la nécessité de normaliser les noms populaires (communs) à l'instar des noms scientifiques ${ }^{2}$ (en acceptant arbitrairement un seul nom par taxon) afin de mettre de l'ordre dans l'impressionnante banque de données terminologiques que constituent les noms botaniques. À titre d'exemple, près de 7000 espèces sont dotées d'un nom français normalisé dans le Elsevier's Dictionary of Trees, Volume 1: North America (Grandtner 2005).

La terminologie proposée dans le présent article a été mise au point pour la publication du dictionnaire de Grandtner (2005). Elle concerne seulement les noms français. Elle s'inscrit dans le prolongement des contributions antérieures (Chancerel 1920; Marie-Victorin 1935; Lamoureux 1975; Fleurbec 1994; Lamoureux 2002 et Blondeau et Roy 2004), en analysant quelques principes novateurs justifiant le choix d'un nom dans un répertoire déjà existant de noms ou ceux justifiant la création de néonymes. Ces principes de normalisation sont les suivants: l'adoption de la forme binomiale (ou trinomiale pour les sous-espèces et les variétés), l'adoption d'un nom botaniquement exact, et le respect des critères d'exclusivité et d'universalité. Bien que le principe d'exclusivité nous oblige à ne retenir qu'un seul nom, donc une seule caractéristique de la plante, il permet d'atteindre un objectif important, celui de faciliter les échanges scientifiques, éducatifs et sociaux (Kelsey et Dayton 1942).

Les principes décrits dans cet article visent à favoriser l'établissement d'une terminologie normalisée française, qui réponde davantage aux critères de normalisation que la terminologie française d'origine purement populaire. Le présent exercice de normalisation a pour objectif de créer, en respectant un cadre formel, de nouveaux noms français lorsqu'ils n'existent pas encore, ce qui était le cas d'environ 80 p. 100 des arbres indigènes d'Amérique du Nord. De plus, nous avons privilégié les noms déjà existants et accordé une priorité aux noms du français standard plutôt qu'aux régionalismes.

Finalement, nous nous pencherons, en détail, à titre d'exemples, sur certaines catégories conceptuelles utilisées pour nommer les arbres, soit la morphologie, la ressemblance, la toponymie, l'écologie, l'anthroponymie et l'utilisation de l'espèce végétale. Les cas des noms d'origine étrangère ou autochtones seront également traités.

Nous nous limiterons aux arbres indigènes d'Amérique du Nord, à l'exclusion des arbres fossiles ou éteints plus récemment, des hybrides et des cultivars. Nous ne traiterons pas, non plus, des noms de leurs fruits, bois ou autres produits. 


\section{Terminologies scientifique, normalisée et populaire}

Dans la présente section, nous abordons trois terminologies, soit la terminologie scientifique latine, la terminologie normalisée française et la terminologie populaire aussi de langue française. Pour illustrer notre propos, nous avons retenu douze espèces (Tableau 1), chacune associée à un nom scientifique, un nom normalisé français et au moins un nom populaire français, dans un échantillon aléatoire de 150 numéros de taxons du dictionnaire des arbres d'Amérique du Nord de Grandtner (2005). Les noms dans ce dictionnaire ont été normalisés selon les principes définis dans la section 3. Les 150 numéros ont été choisis au hasard au moyen de la fonction statistique [=alea.entre.bornes $(1 ; 8778)]$ du logiciel Excel, puis triés par ordre croissant et reclassés par ordre alphabétique au moyen du logiciel de traitement de texte Word, ce qui a finalement permis une analyse comparative de chaque terminologie.

TABLEAU 1

Les douze espèces arborescentes sélectionnées et leurs dénominations selon les terminologies scientifique, normalisée et populaire

\begin{tabular}{|c|c|c|c|}
\hline $\mathbf{N}^{\circ}$ & Nom scientifique latin & Nom normalisé français & $\operatorname{Nom}(s)$ populaire(s) français \\
\hline 1. & Bursera microphylla & gommier à petites feuilles & gomart à petites feuilles \\
\hline 2. & Carya laciniosa & caryer lacinié & $\begin{array}{l}\text { carya lacinié, carya à écorce } \\
\text { laciniée, caryer à folioles } \\
\text { denticulées, hickory écailleux }\end{array}$ \\
\hline 3. & Coccoloba pubescens & raisinier grandes-feuilles & $\begin{array}{l}\text { bois grandes-feuilles, gamelle, } \\
\text { grande-feuille, magne la mer, } \\
\text { moralón, raisin grandes-feuilles, } \\
\text { raisin marron, } \\
\text { raisinier-montagne }\end{array}$ \\
\hline 4. & Cordia collococa & cordia mapou-rivière & $\begin{array}{l}\text { mahot bré, mahot rivière, mapou } \\
\text { mapou blanc, mapou rivière } \\
\text { trois pieds }\end{array}$ \\
\hline 5. & Diospyros revoluta & plaqueminier barbaquois & $\begin{array}{l}\text { barbacoa, barbacoar, barbacois } \\
\text { bois négresse, bois négresse } \\
\text { ébène, bois noir, ébène } \\
\text { plaqueminier }\end{array}$ \\
\hline 6. & Diospyros tetrasperma & plaqueminier à quatre graines & bois raide \\
\hline 7. & Hypelate trifoliata & gallipeau trifolié & chandelle marron, gallipeau \\
\hline 8. & Meliosma abbreviata & méliosma raccourci & coma, coma jaune \\
\hline 9. & Myrcianthes fragrans & myrcianthes fragrant & $\begin{array}{l}\text { bois créole, bois d'inde, bois } \\
\text { d'inde bâtard, bois d'inde bois } \\
\text { haut-goût, bois mulâtre, bois pelé, } \\
\text { eugénie à fruits géminés, eugénie } \\
\text { de simpson, goyavier bois, } \\
\text { goyavier montagne, mérisier, } \\
\text { myrte à feuilles de laurier }\end{array}$ \\
\hline 10. & Podocarpus aristulatus & podocarpe aristé & bois lubin, cœur noir \\
\hline 11. & Psychotria pubescens & ramier pubescent & café marron \\
\hline 12. & Talauma dodecapetala & talauma bois-pain & $\begin{array}{l}\text { bois-pin, bois-pain marron, } \\
\text { cachiman-montagne, magnolia }\end{array}$ \\
\hline
\end{tabular}


En portant une attention aux épithètes (ou déterminants) spécifiques des noms scientifiques et des noms normalisés français, nous constatons que cinq des douze noms scientifiques ont la même origine étymologique que leur équivalent normalisé:

Carya laciniosa (caryer lacinié)

Hypelate trifoliata (gallipeau trifolié)

Myrcianthes fragrans (myrcianthes fragrant)

Podocarpus aristulatus (podocarpe aristé)

Psychotria pubescens (ramier pubescent)

En revanche, en comparant la terminologie scientifique à la terminologie populaire française, et toujours en portant notre attention aux épithètes, nous constatons qu'aucun des noms populaires français n'a la même origine étymologique que le nom scientifique correspondant.

En portant maintenant notre attention à la conservation du sens des épithètes, nous pouvons ajouter les trois noms scientifiques suivants et leur équivalent normalisé:

Bursera microphylla (gommier à petites feuilles)

Diospyros tetrasperma (plaqueminier à quatre graines)

Meliosma abbreviata (méliosma raccourci)

Nous pouvons donc conclure, après le traitement de normalisation de Grandtner (2005), qu'il arrive souvent que les noms normalisés français partagent leur origine étymologique avec le nom scientifique, ou du moins, que le sens du nom normalisé français et du nom scientifique coïncident. Si nous comparons maintenant les noms scientifiques avec les noms populaires français, nous observons souvent une plus grande disparité dans le sens des équivalents (ou variantes).

Analysons maintenant les forces et les faiblesses de chaque terminologie. La première terminologie (terminologie scientifique latine) se fonde sur un réseau notionnel bien hiérarchisé (taxonomie). Ce réseau comprend des centaines de milliers de termes et son pouvoir classificatoire fait l'envie de bien d'autres terminologies plus récentes (Otman 1996). Pour bien comprendre et bien savoir utiliser cette terminologie, et par extension la taxonomie de référence, de nombreuses années d'études sont nécessaires. De plus, comme cette terminologie a une forme latine, elle n'est certes pas à la portée de tous. À l'autre extrême, la terminologie populaire française est beaucoup plus accessible. Elle a souvent été élaborée localement pour nommer les espèces propres à une zone géographique plus ou moins étendue (la province de Québec par exemple), mais elle a ses limites. En effet, ne disposant pas d'un réseau notionnel aussi vaste et précis que celui de la taxonomie, sa capacité de nommer les espèces et de les catégoriser est plus faible. Elle peut convenir pour nommer un certain nombre d'espèces; cependant, lorsque ce nombre devient plus important, l'entropie du système de nomenclature populaire augmente beaucoup plus rapidement que l'entropie du système de nomenclature scientifique (attribuable entre autres à la synonymie). Considérons maintenant une troisième terminologie, la terminologie normalisée française. La terminologie normalisée française se fonde en grande partie sur la terminologie scientifique. Elle repose sur le même grand réseau notionnel, la taxonomie, à la différence qu'on utilise le français pour nommer les espèces. Il existe donc une coïncidence conceptuelle avec la terminologie latine (voir le tableau 1). Cette terminologie regroupe en quelque sorte les avantages de la terminologie scientifique et de la terminologie populaire française. Pour tout projet ambitieux visant à 
nommer un grand nombre d'espèces en français, elle s'avère l'option idéale; elle permet en effet l'élaboration d'une nomenclature de langue française en gardant la très grande capacité d'organisation de la taxonomie surtout exprimée en latin dans le passé.

\section{Principes de normalisation}

La normalisation doit ici être comprise comme un acte externe d'uniformisation terminologique réalisé par des spécialistes du domaine ${ }^{3}$ et qui va privilégier l'usage d'un terme unique parmi un ensemble de variantes terminologiques (Cabré 1998). Elle s'applique aussi, bien souvent, à l'uniformisation des procédés de formation terminologique disponibles en français moderne (Duquet Picard 1985). Selon nous, quatre principes fondamentaux devraient régir ce processus: le caractère binomial (ou trinomial), le caractère exclusif, l'exactitude de la signification botanique et l'universalité du nom.

\subsection{Caractère binomial ou trinomial du nom}

Le nom normalisé d'une espèce doit toujours comprendre deux éléments. Un nom générique ('sapin', pour Abies) et une épithète spécifique ('baumier', pour balsamea). Le nom français normalisé de l'Abies balsamea sera donc 'sapin baumier'. En outre, le nom du genre doit être normalement le même pour toutes ses espèces, sauf de rares exceptions où l'usage impose des noms différents ('abricotier', 'amandier', 'cerisier', 'pêcher' et 'prunier', appartenant tous au genre Prunus) ${ }^{4}$. C'est le contraire pour l'épithète spécifique, qui doit être différente pour chaque espèce d'un même genre. Il en est de même pour les sous-espèces et les variétés, dont le nom devient trinomial. Lorsque l'épithète scientifique de ces dernières est identique à l'épithète de l'unité taxonomique immédiatement supérieure, on doit les qualifier de «typique» (Amelanchier arborea var. arborea devient 'amélanchier arborescent typique').

\subsection{Exclusivité}

Le nom normalisé doit également être exclusif. Il doit s'appliquer à un seul taxon, c'est-à-dire à une seule unité systématique de classification (genre, espèce, sousespèce ou variété aux fins du présent article). Dans le cas du genre, le nom 'cèdre', par exemple, doit désigner uniquement les espèces du genre Cedrus et non aussi celles du genre Cedrela ('cédrela'), Cupressus ('cyprès'), Juniperus ('genévrier'), Nectandra ('nectandra') et Thuja ('thuya'). La même épithète spécifique peut cependant servir à nommer plusieurs espèces appartenant à des genres différents ('cerisier de la Virginie' [Prunus virginiana], 'chêne de la Virginie' [Quercus virginiana], 'pin de la Virginie’ [Pinus virginiana], etc.). Ce qui doit finalement être exclusif dans le cas du nom d'une espèce, c'est sa combinaison binomiale, c'est-à-dire l'association d'un nom de genre avec une épithète spécifique.

\subsection{Exactitude de la signification botanique}

Le processus de normalisation doit aboutir à la formation d'un nom exact sur le plan botanique. On ne peut pas nommer 'cyprès' (Cupressus) le pin gris (Pinus banksiana), 
ni 'épinette rouge' (Picea rubens) le mélèze laricin (Larix laricina), ni 'merisier' (Prunus) le bouleau jaune (Betula alleghaniensis), ni 'noyer' (Juglans) un caryer (Carya) ou un clavalier (Zanthoxylum).

\subsection{Universalité}

Le nom normalisé devrait être universel plutôt que régional, c'est-à-dire le même partout. On devrait donc retenir 'épicéa' (Picea) de préférence à 'épinette', et 'tsuga' (Tsuga) au lieu de 'pruche' comme proposé par Chancerel (1920), Marie-Victorin (1935) et Grandtner (2005). Procéder autrement risquerait de provoquer une multiplication indue des noms génériques normalisés utilisés pour un même genre et de contrevenir ainsi au principe du nom binomial, qui exige que, normalement, le nom binomial de toutes les espèces d'un même genre commence par le même nom générique.

En plus de ces quatre principes de base, la normalisation des noms botaniques français devrait obéir aux principes suivants.

\subsection{Choix d'un nom existant ou néonyme?}

Si un nom populaire conforme aux quatre principes précités existe déjà, il devrait être retenu comme nom normalisé, en priorité. Dans le cas contraire, il devrait être remplacé par un néonyme. Ce dernier peut être, selon Hermans et Vansteelandt (1999) soit primaire, lorsque le concept n'existe pas encore, soit traductif (secondaire), lorsque le concept existe déjà dans une langue et qu'il est traduit dans une autre langue. Le néonyme peut être attribué à l'un ou l'autre élément du nom binomial, ou aux deux à la fois.

Pour ce qui est des deux types évoqués de néologismes, les procédés suivants peuvent être utilisés.

\subsubsection{Traduction et néologismes primaires}

La traduction du latin représente le procédé le plus fréquent de la néologie botanique, bien que la traduction à partir d'une autre langue de départ, par exemple à partir de l'anglais, existe également, surtout en Amérique du Nord. Ce procédé a cependant un inconvénient. En effet, à la suite de changements apportés au nom latin (qui se produisent malheureusement souvent), le néonyme issu d'une traduction devrait changer à son tour, ce qui a pour effet de provoquer une certaine instabilité dans le système de nomenclature normalisée ${ }^{5}$.

Chez les arbres d'Amérique du Nord (Grandtner 2005), comme chez ceux du Québec examinés récemment par Auger (2008), les appellations latines et les néologismes traductifs et primaires se rapportent à une de leurs caractéristiques morphologiques (noms morphologiques), à leur ressemblance avec un autre arbre (noms attribuables à la ressemblance), à la répartition géographique (noms toponymiques), à l'habitat (noms écologiques), à des noms de personnes ou de peuples (noms anthroponymiques), ou à l'usage que l'on fait de l'arbre (noms reliés à l'utilisation de l'espèce).

C'est sur ce plan surtout, mais non exclusivement, que se situe l'intérêt d'une collaboration entre un spécialiste des arbres, voire des plantes en général, et un spécialiste 
de la langue, évoqué par Duquet Picard (1985) et illustré par Bizet et Walter (2008) et par le présent article.

\subsubsection{Noms morphologiques}

Les noms liés aux caractéristiques morphologiques constituent la catégorie la plus utilisée et concernent surtout la taille, la forme, la présence ou absence d'épines ou de pilosité, ainsi que la couleur de l'arbre ou d'une de ses parties. Par exemple, le séquoiadendron géant (Sequoiadendron giganteum) est un arbre qui peut mesurer plus de $100 \mathrm{~m}$ de hauteur et $10 \mathrm{~m}$ de diamètre, alors que le nom du licania à petites fleurs (Licania micrantha) fait référence à la petite taille de ses fleurs. Pour sa part, la forme obtuse des feuilles justifie l'appellation du jacaranda à feuilles obtuses (Jacaranda obtusifolia), alors que la présence ou l'absence d'épines expliquent celles du cordia épineux (Cordia spinescens) et du cordia inerme (Cordia inermis). Des poils sont évidemment présents chez le bunchosia poilu (Bunchosia pilosa). Enfin, le nom du bouleau jaune (Betula alleghaniensis) est lié à la couleur de son écorce.

\subsubsection{Noms associés à une ressemblance}

Lorsqu'un arbre ressemble à un autre taxon, son épithète spécifique peut contenir le nom de ce dernier, accompagné du préfixe «faux-». Par exemple, le peuplier fauxtremble (Populus tremuloides), une espèce nord-américaine de peuplier qui ressemble au peuplier tremble (Populus tremula) d'Europe. Lorsqu'il s'agit de la ressemblance d'une partie de l'arbre, il faut la préciser, comme dans le cas du figuier à feuilles de fustet (Ficus cotonifolia).

\subsubsection{Noms toponymiques}

De la même façon que les épithètes toponymiques latines, traitées par Kunkel (1990) et Beaulieu (2005), les épithètes françaises normalisées peuvent être dérivées de l'appellation d'un continent ou de ses divisions géographiques, politiques, orographiques ou hydrographiques. Elles expriment, essentiellement, la répartition du taxon, ou le lieu de récolte du spécimen-type ayant servi à sa description. Les exemples suivants illustrent les principales catégories de toponymes utilisés.

(1) Divisions géographiques

- région: Juniperus occidentalis (genévrier occidental), taxon présent dans l'ouest du continent nord-américain, ou plus exactement des États-Unis d'Amérique, depuis les États de Washington et d'Idaho jusqu'à l'État de Californie;

- péninsule: Caesalpinia yucatanensis (césalpinie du Yucatán), présente notamment au Mexique, dans la péninsule du Yucatán;

- isthme: Rudgea isthmensis (rudgéa de l'isthme), présent dans l'isthme du Panamá;

- archipel ou île: Quercus tomentella (chêne insulaire), présent dans les îles Channel de Californie et dans l'île Guadalupe de Basse-Californie.

(2) Divisions politiques

- pays: Amyris guatemalensis (amyris du Guatemala);

- État: Pinus jaliscana (pin du Jalisco), le Jalisco étant un État du Mexique central;

- province: Crataegus pedicellata (aubépine de l'Ontario), traduction de son nom anglais Ontario hawthorn; 
- localité: Clusia palmana (clusia de La Palma), lieu de récolte du spécimen-type de ce taxon au Costa Rica.

(3) Divisions orographiques

- montagne: Psychotria tacarcunensis (ramier du Cerro Tacarcuna), le spécimentype de ce taxon a été récolté dans le massif du Cerro Tacarcuna, au Panamá;

- colline: Psychotria clivorum (ramier des collines), traduit du latin;

- vallée: Trophis caucana (ramón du Cauca), récolté dans la vallée du Cauca, en Colombie.

(4) Divisions hydrographiques

- océan: Magnolia pacifica (magnolia du Pacifique), présent sur la côte du Pacifique, dans l'État du Jalisco, au Mexique;

- autres divisions: bassin hydrographique, rivière, lac, etc.

L'utilisation des noms toponymiques est particulièrement fréquente pour les arbres endémiques au sens strict, c'est-à-dire à répartition très restreinte, limitée à un seul pays, État, province ou archipel, voire à une seule île ou montagne. Par exemple, pour le Clusia domingensis, nous avons utilisé de préférence le nom «clusier d'Hispaniola», car l'espèce est endémique de l'île dans son ensemble et non seulement de République dominicaine.

\subsubsection{Noms écologiques}

Par opposition aux noms toponymiques, dictés par la géographie, les noms écologiques expriment la préférence d'un arbre pour un type de milieu. Ces noms sont dérivés d'un nom latin ou grec du facteur physique, chimique ou biologique prédominant dans un milieu donné. Les principaux facteurs sont décrits dans Grandtner, Chevrette, et al. (2007). En voici des exemples:

(1) Lumière

- On qualifie de héliophiles, ou intolérants à l'ombre, les arbres qui poussent bien en pleine lumière, tels que le lonchocarpe rugueux héliophile (Lonchocarpus rugosus subsp. apricus). À l'opposé de cela se trouvent des arbres sciaphiles, ou tolérants à l'ombre, qui poussent bien dans un milieu ombragé.

(2) Eau du sol

- On qualifie de hygrophiles les arbres qui poussent bien dans des sols humides ou inondés pendant une période plus ou moins longue, de mésophiles ceux qui poussent bien dans des sols d'humidité moyenne et, enfin, de xérophiles ceux qui poussent bien dans des sols secs, comme l'eugénie xérophile (Eugenia xerophytica).

(3) Réaction du sol

- Selon le degré d'acidité ou d'alcalinité du sol, les arbres peuvent se nommer acidophiles, quand ils poussent bien en sols acides $(\mathrm{pH}<6,5)$, ou neutrophiles ( $\mathrm{pH} 6,5$ $7,5)$, ou basiphiles $(\mathrm{pH}>7,5)$.

(4) Substrat

- Les arbres qui poussent bien sur des substrats calcaires, comme l'inga calcicole (Inga calcicola), sont qualifiés de calcicoles, alors que ceux qui poussent bien sur des substrats serpentineux, comme le tabébuia serpentinicole (Tabebuia ophiolitica), sont appelés serpentinicoles. Enfin, ceux qui poussent bien sur des substrats siliceux portent le nom de silicicoles. 
(5) Type de milieu

- Les arbres peuvent, par ailleurs, recevoir une épithète dérivée du nom d'un type particulier de milieu où ils réussissent à bien pousser, par exemple le marécage, comme dans le cas du jacquinier paludicole (Jacquinia paludicola), ou le désert, dans celui du chêne du désert (Quercus deserticola).

\subsubsection{Noms anthroponymiques}

Ces noms sont dérivés de noms ou prénoms de personnes, ou de noms de peuples, que les botanistes décrivant un nouveau taxon désiraient honorer; par exemple, le cratéva de Palmer (Crateva palmeri) et l'acacia des Mayas (Acacia mayana). Souvent, il s'agit du nom de la personne qui a récolté le spécimen type ayant servi à la description du nouveau taxon, comme dans le cas de l'aubépine de Victorin (Crataegus victorinii), ou de l'eugénie de Holdridge (Eugenia holdridgei).

\subsubsection{Noms liés à l'utilisation}

Les noms liés à l'utilisation de l'arbre peuvent être dérivés du nom d'un constituant alimentaire ou médicinal, ou d'une autre substance quelconque de l'arbre. L'un des constituants alimentaires le mieux connu en Amérique du Nord est le sucre d'érable, présent notamment dans la sève de l'érable à sucre (Acer saccharum). D'autres produits qui ont donné leur nom à des arbres sont décrits dans Grandtner, Chevrette, et al. (2007). Ce sont surtout des substances médicinales telles que tanins médicaux, gommes pharmaceutiques et cosmétiques, insecticides, parfums, etc., dont la saponine utilisée pour les savons et que l'on trouve, par exemple, dans les feuilles et les racines du savonnier des Antilles (Sapindus saponaria).

\subsubsection{Autres procédés}

\subsubsection{Noms d'origine étrangère}

La langue d'origine la plus fréquemment utilisée, notamment pour les noms de genre, est le latin. Lorsque l'équivalent français existe déjà, il doit être retenu en priorité (p. ex., 'bacchante' pour Baccharis). S'il n'existe pas encore et que sa francisation est possible, on doit l'effectuer (p. ex., 'beaucarnéa' pour Beaucarnea). Enfin, si la francisation n'est pas possible, on doit garder le nom tel qu'il existe en latin (p. ex., 'bactris' pour Bactris). Le même procédé est appliqué aux autres langues et aux épithètes spécifiques et infraspécifiques.

\subsubsection{Noms autochtones}

Plusieurs noms scientifiques sont dérivés de noms autochtones auxquels on a appliqué le procédé décrit au paragraphe précédent. On retiendra, par exemple, jacaranda pour Jacaranda, nom guarani que le français retient tel quel, ou eugénie capuli (Eugenia capuli), de la langue nahuatl.

\section{Conclusion}

La désignation des plantes est une préoccupation très ancienne, en grande partie liée au fait que le nom attribué à une plante ne peut évoquer qu'une de ses nombreuses caractéristiques: un nom, un concept, une espèce. C'est pourquoi la terminologie populaire aboutit à une multiplication débridée des noms. 
Pour pallier ce problème, il est important de créer une terminologie française normalisée, ayant des assises solides dans la taxonomie, si l'on doit, par exemple, nommer un vaste répertoire d'arbres. Afin de réduire l'entropie du système, une telle terminologie se doit d'être strictement binomiale (avec un seul nom générique pour nommer des espèces appartenant au même genre), exclusive, botaniquement exacte et universelle. Dans le présent article, nous examinons six grandes catégories conceptuelles pouvant nous guider pour le choix des noms botaniques: la morphologie, la ressemblance de l'espèce, la toponymie, l'écologie, l'anthroponymie et l'utilisation de la plante. Il va sans dire que d'autres renseignements de nature définitoire ou contextuelle peuvent être utilisés pour compléter l'information véhiculée par le nom.

Selon nos estimations, la dendroflore mondiale compterait près de 60000 taxons. Environ 80 p. 100 de ceux-ci n’ont pas encore de nom français. Nommer en français la grande majorité des arbres, selon les principes de normalisation proposés dans le présent article, permettrait de stocker, pour le monde francophone, des informations primordiales sur la diversité de la vie, laquelle diversité est entre autres menacée par les changements climatiques (Grandtner 2006) et la destruction des forêts tropicales (Beaulieu 2006).

Cette nomenclature normalisée française assurerait une plus grande uniformité des noms français, ce qui faciliterait les échanges et stimulerait l'activité scientifique, socio-économique et culturelle. Enfin, un tel effort permettrait de vivre davantage en harmonie avec la dendroflore et de connaître un peu mieux, sans risque de confusion, chaque arbre (voire, par extension, chaque plante) poussant sur notre planète.

\section{REMERCIEMENTS}

Nous aimerions remercier tout particulièrement Pierre Auger, Marc Favreau, Gabriel Huard et les deux évaluateurs anonymes pour leurs suggestions constructives, ainsi que Stéphane Plante pour les méthodes statistiques et Dominique Garneau pour le traitement du texte.

\section{NOTES}

1. Les opinions exprimées dans le présent article n'engagent que ses auteurs et ne sauraient être considérées comme l'expression du point de vue du gouvernement du Canada.

2. La force de la nomenclature latine scientifique et de son pouvoir de normalisation vient principalement du principe de la priorité de la publication des noms botaniques (McNeill 2006). Par extension, nous proposons dans le présent article que ce principe pourrait s'appliquer aux langues vernaculaires et, en l'occurrence, au français. De plus, nous nous sommes inspirés des règles et recommandations du Code international de botanique (McNeill 2006) pour l'élaboration des autres principes de normalisation. Ces principes pourraient représenter une piste de travail pour toute autorité chargée de gérer les noms botaniques dans le monde francophone.

3. Le travail de normalisation dans le dictionnaire de Grandtner (2005) a été effectué pour les noms français et anglais. Il est important de noter que la normalisation ne peut se faire de la même façon d'une langue à l'autre. Une étude par corpus de Beaulieu (2009), se penchant sur les épithètes toponymiques, révèle d'ailleurs que les noms français sont plus intimement liés à la taxonomie que les noms anglais ou espagnols.

4. Peut-être serait-il avantageux, dans l'intérêt de la stabilité d'une terminologie normalisée, de prévoir, dans le cas où il existe plusieurs classifications, que la terminologie normalisée soit basée en priorité sur la taxonomie reconnue la plus récente.

5. Pour cette raison, il serait essentiel de promouvoir l'indépendance des termes français de la nomenclature scientifique latine (c'est-à-dire sa stabilité par rapport aux changements nomenclaturaux qui ne seraient pas fondés sur une notion modifiée des espèces et des genres). En revanche, il faudrait encourager leur dépendance à des changements d'ordre taxonomique. 


\section{RÉFÉRENCES}

Auger, Pierre (2008): Les termes complexes dans les dendronymes québécois. Onomastica Canadiana. 90(1):1-19.

Beaulieu, Marc-Alexandre (2005): L'onomastique en botanique. Onomastica Canadiana. 87(2):55-70.

Beaulieu, Marc-Alexandre (2006): L'onomastique en botanique: un survol de quatre pays d'Amérique du Sud. Onomastica Canadiana. 88(2):19-39.

Beaulieu, Marc-Alexandre (2009): Traduction multilingue de toponymes en botanique. Names in Multi-Lingual, Multi-Cultural and Multi-Ethnic Contact. Proceedings of the $23^{\text {rd }}$ International Congress of Onomastic Sciences. York University, Toronto, Canada, 17-22 août.

Bizet, Ange et Walter, Annie (2008): Problématique de terminologie botanique en français: l'exemple des fruits, noix et arbres fruitiers du Vanuatu (Vanouatou). La banque des mots. 76:31-40.

Blondeau, Marcel et Roy, Claude (2004): Atlas des plantes des villages du Nunavik. Sainte-Foy: Multimondes.

CABré, Maria Teresa (1998): La terminologie. Théorie, méthode et applications. Ottawa: Presses de l'Université d'Ottawa et Paris: Armand Colin.

Chancerel, Lucien (1920): Flore forestière du globe. Paris: Gauthier-Villars.

Duquet PiCard, Diane (1985): Normalisation et néonymie en français moderne. Meta 30(3):257-260.

Fleurbec/Lamoureux, Gisèle, Lamoureux, Sylvain, Tousignant, Aude, Cournoyer, Luc et Gauthier, Robert F. (1994): Plantes susceptibles d'être menacées ou vulnérables. Noms français de 229 espèces. Rapport non publié, préparé pour le gouvernement du Québec. Québec: Ministère de l'Environnement et de la Faune, Direction de la conservation et du patrimoine écologique.

Grandtner, Miroslav M. (2005): Elsevier's Dictionary of Trees. Volume 1: North America. Amsterdam: Elsevier.

Grandtner, Miroslav M. (2006): North American Native Tree Species Diversity. Polish Botanical Studies. 22:207-210.

Grandtner, Miroslav M., Chevrette, Julien, des Rochers, Renée, Gagné, France et Garneau, Dominic (2007): Dictionnaire mondial des arbres. Instructions générales aux collaborateurs. $9^{\mathrm{e}}$ approximation. Québec: Département des sciences du bois et de la forêt, Faculté de foresterie et de géomatique, Université Laval.

Hermans, Adrien et Vansteelandt, Andrée (1999): Néologie traductive. Terminologies nouvelles 20:37-43.

Kelsey, Harlan P. et Dayton, William A. (1942): Standardized Plant Names. $2^{\mathrm{e}}$ éd., Harrisburg: McFarland.

Kunkel, Günther (1990): Geography Through Botany: A Dictionary of Plant Names with a Geographical Meaning. The Hague: Academic Publishing.

Lamoureux, Gisèle (2002): Flore printanière. Saint-Henri-de-Lévis: Fleurbec.

Linnaeus, Carolus (2003): Philosophia Botanica. (Translated by Stephen Freer). New York: Oxford University Press.

Marie-Victorin, Frère (1935): Flore laurentienne. Montréal: Les Frères des écoles chrétiennes.

McNeILL, John (2006): International Code of Botanical Nomenclature (Vienna Code) adopted by the Seventeenth International Botanical Congress. Vienna, Austria.

Otman, Gabriel (1996): Les représentations sémantiques en terminologie. Paris: Masson.

Pajaud, Daniel (1989): Nomenclature et taxinomie. Pratique de la dénomination et usage des concepts en ontologie. Paris: Gaston Lachurié. 